\title{
Diagnosing Meat Allergy After Tick Bite Without Delay
}

\author{
Adam C. Kaplan, MD, and Michael P. Carson, MD
}

Galactose- $\alpha-1,3$ galactose ( $\alpha$-gal) is a carbohydrate found in mammalian meat. In 2007, it was implicated as a cause of severe hypersensitivity reactions when a study found elevated levels of antibodies directed against this oligosaccharide among patients treated with cetuximab, a monoclonal antibody that contained an $\alpha$-gal epitope. The majority of these cases were reported in the Southeast United States in a distribution similar to that of Rocky Mountain spotted fever and ehrlichiosis, and that geographic association led researchers to the conclusion that a bite from the Lone Star tick can induce this antibody. Here, we present a case of delayed urticarial angioedema due to a mammalian meat allergy caused by $\alpha$-gal immunoglobulin $E$ acquired after tick exposures, and the knowledge and patient education required to prevent recurrences. It is estimated that approximately $0.5 \%$ to $1.0 \%$ of the general population will experience an episode of angioedema in their lifetime, and this case demonstrates why clinicians in areas that are inhabited by ticks, particularly the Lone Star species, should include this cause in their differential. ( $\mathrm{J}$ Am Board Fam Med 2018;31:650-652.)

Keywords: Angioedema, Cetuximab, Food Hypersensitivity, Galactosyl-(1-3)galactose, Meat, Southeastern United States, Tick Bites

Angioedema involves swelling of the deep dermal, subcutaneous, or submucosal tissue due to vascular leakage that can be life-threatening and recurrent if the trigger is not identified. We present a case of delayed angioedema due to a mammalian meat allergy caused by antigalactose- $\alpha-1,3$ galactose-immunoglobulin $\mathrm{E}(\mathrm{IgE})$ antibodies ( $\alpha$-gal) acquired after tick exposures, along with the measures needed to prevent recurrence. Recent evidence shows that bites from the Lone Star tick (Amblyomma americanum) can induce this antibody. ${ }^{1} \mathrm{Ap}$ proximately $0.5 \%$ to $1.0 \%$ of the general population will experience an episode of angioedema in their lifetime, and this case demonstrates why clinicians in areas that are inhabited by ticks, partic-

This article was externally peer reviewed.

Submitted 31 October 2017; revised 1 March 2018; accepted 3 March 2018.

From Jersey Shore University Medical Center, Neptune, NJ (ACK, MPC).

Funding: none.

Conflict of interest: none declared.

Corresponding author: Adam C. Kaplan, MD, Jersey Shore University Medical Center, Neptune, NJ (E-mail: Adam. Kaplan@hackensackmeridian.org). ularly the Lone Star tick, ${ }^{*}$ should include this cause in their differential. ${ }^{2}$

The objective of this study was to recognize the etiology, signs, symptoms, and prevention of $\alpha$-gal allergy, along with the potential for increasing prevalence in geographic areas with known Lone Star tick habitation.

A 43-year-old woman with no past medical history was camping in Pennsylvania. The night of symptom onset, she had a dinner that included grilled steak. At 2 am, she awoke feeling "unwell" and developed diarrhea, vomiting, severe itching, generalized hives, and swelling of her extremities and face. The symptoms improved with diphenhydramine. Three days later, she saw an allergist who ascertained that a week before, after a hike in Monmouth County, NJ, she removed multiple ticks of unknown species, but reported no symptoms. After those tick exposures, the first time she had red meat was the night of symptom onset. A physical exam-

\footnotetext{
*To see a map showing the approximate distribution of the Lone Star tick, visit the Centers for Disease Control and Prevention website at https://www.cdc.gov/ticks/maps/lone star_tick.html.
} 
ination at the office visit revealed normal vital signs and no systemic signs of illness, including rashes, organomegaly, or edema. Immunologic testing supported the clinical diagnosis of $\alpha$-gal allergy syndrome: beef-specific IgE levels, $1.39 \mathrm{kU} / \mathrm{L}$ $(<0.35 \mathrm{kU} / \mathrm{L})$; pork, $0.83 \mathrm{kU} / \mathrm{L}(<0.35 \mathrm{kU} / \mathrm{L})$; lamb, $0.64(<0.35 \mathrm{kU} / \mathrm{L})$; as well as $\alpha$-gal $\operatorname{IgE}, 2.41$ $(<0.35)$. She was instructed to avoid ingestion of the $\alpha$-gal carbohydrate, which is found naturally in mammalian meats, and an epinephrine pen was provided for emergency use.

Following the diagnosis, she avoided mammalian meat, but hives, diarrhea, and swelling continued to reoccur. Vigilance with adhering to a mammalian-free diet and avoiding other potential mammalian products such as gelatin and dairy was associated with a decrease in the severity and frequency of her episodes over a 10 -month period.

\section{Discussion}

This case highlights the importance of recognizing the history, signs, and symptoms consistent with an $\alpha$-gal allergy. Allergic reactions to $\alpha$-gal were first described in cancer patients treated with the monoclonal antibody cetuximab, which contained the $\alpha$-gal epitope. However recent data shows that tick bites, particularly from the Lone Star species, can induce this antibody. ${ }^{1}$ The reasons certain patients develop intolerance, the exact time frame of allergy sensitization, and if our patient was sensitized by her recent exposure versus an earlier one remain uncertain. The syndrome is caused by sensitization to this oligosaccharide in predisposed patients. ${ }^{3}$ The role of ticks, particularly the Lone Star Tick, is less well understood, but a cross-sectional study found that $97 \%(117 / 121)$ of patients presenting to an allergy clinic with history of delayed anaphylaxis to red meat had anti- $\alpha$-gal $\operatorname{IgE}$, compared with only $11 \%(6 / 56)$ of patients seen in that same practice for asthma. ${ }^{1}$ In addition, the prevalence of $\alpha$-gal antibodies was $20 \%$ in tick-prone regions (Tennessee, North Carolina, and Virginia) compared with $<1 \%$ in areas without ticks, including Boston, Sweden, or North Carolina. This distribution was similar to the distribution of allergy reports associated with cetuximab administration and is how the original link between cetuximab, the Lone Star tick, and $\alpha$-gal were connected. Within this study, prospective data on 3 patients showed a 20 -fold increase in serum titers from baseline of anti- $\alpha$-gal $\operatorname{IgE}$ antibodies after a tick bite, and 2 of these 3 patients experienced a hypersensitivity reaction after ingesting mammalian products. Although the reaction to $\alpha$-gal antibodies is commonly referred to as a "delayed anaphylactic reaction," it should be noted that it is truly an $\mathrm{IgE}$-mediated reaction that can result in life-threating acute anaphylaxis. Use of the term "delay" in the description refers to the time from ingestion of the meat to symptom onset, which is typically 4 hours. This delay can result in prolonging the time to diagnosis, as patients may not perceive a correlation between the symptoms and ingestion of the offending agent hours before. Therefore, expanding physician awareness of this syndrome will prompt them to obtain a detailed dietary history contemporaneous to the time of symptom onset. Adding to the complexity of this diagnosis, patients may have a negative reaction to skin prick allergy testing as well. ${ }^{4}$

Maintaining a high index of suspicion for $\alpha$-gal allergy in areas with a high prevalence of ticks, particularly the Lone Star tick, and the aforementioned symptoms is of paramount importance, as establishing the diagnosis can be lifesaving. Current prevention is aimed at tick avoidance, including long sleeves/pants and tick repellants. Once diagnosed, a patient's treatment should be aimed at preventing exposure by avoidance of mammalian meat ingestion and, in cases such as ours, consider restriction of products, such as gelatin and dairy that may be derived from mammalian sources. ${ }^{5}$ The long-term prognosis of this condition is not well described; however, it is thought that if a strict diet is followed and tick re-exposure is avoided, reactions may lessen over time. ${ }^{6}$

A better understanding of this delayed immediate hypersensitivity reaction through further research is essential for providing optimal care to patients who have become sensitized, including identifying other vectors due to symptoms seen in Europe and Australia where the Lone Star Tick is not endemic, elucidating the natural time frame for this allergy, and clinical management to help guide avoidance. Finally, we need to identify potential triggers for a reaction and their implications for medical treatment, such as implantation of artificial porcine heart valves, grafts, and even use of bovine/ porcine heparin products. ${ }^{7}$

Educating physicians and patients about the distribution of the Lone Star tick, the delayed allergy 
response, the constellation of symptoms (gastrointestinal symptoms, hives, angioedema, and anaphylaxis), and how to protect oneself with repellents, clothing, and skin checks is of the utmost importance. Once the allergy is identified or suspected, the first steps should include patient education regarding avoidance of mammalian meat products, prescription of an autoinjector epinephrine pen, and due to the many unknowns about the management and course of this allergy, referral to an allergist should be considered.

To see this article online, please go to: http://jabfm.org/content/ 31/4/650.full.

\section{References}

1. Commins SP, James HR, Kelly LA, et al. The relevance of tick bites to the production of $\mathrm{IgE}$ antibodies to the mammalian oligosaccharide galactose-alpha-1,3-galactose. J Allergy Clin Immunol. 2011; 127:1286-93 e6.

2. Mansi M, Zanichelli A, Coerezza A, et al. Presentation, diagnosis and treatment of angioedema without wheals: a retrospective analysis of a cohort of 1058 patients. J Intern Med 2015;277:585-93.

3. Saleh H, Embry S, Nauli A, Atyia S, Krishnaswamy G. Anaphylactic reactions to oligosaccharides in red meat: a syndrome in evolution. Clin Mol Allergy 2012;10:5.

4. Wolver SE, Sun DR, Commins SP, Schwartz LB. A peculiar cause of anaphylaxis: no more steak? The journey to discovery of a newly recognized allergy to galactose-alpha-1,3-galactose found in mammalian meat. J Gen Intern Med 2013;28:322-5.

5. Commins SP, Jerath MR, Cox K, Erickson LD, Platts-Mills T. Delayed anaphylaxis to alpha-gal, an oligosaccharide in mammalian meat. Allergol Int 2016;65:16-20.

6. Fuentes Aparicio V, Sanchez Marcen I, Perez Montero A, Baeza ML, de Barrio Fernandez M. Allergy to mammal's meat in adult life: immunologic and follow-up study. J Investig Allergol Clin Immunol 2005;15:228-31.

7. Kleiman AM, Littlewood KE, Groves DS. Delayed Anaphylaxis to mammalian meat following tick exposure and its impact on anesthetic management for cardiac surgery: a case report. A A Case Rep 2017; $8: 175-7$. 